\title{
Transient acute-onset tetraparesis in a COVID-19 patient
}

\author{
This article has been corrected since Advance Online Publication and a correction is also printed in this issue
}

\author{
Andrea Giorgianni ${ }^{1} \cdot$ Gabriele Vinacci ${ }^{2}$ Edoardo Agosti $\mathbb{C}^{3} \cdot$ Lucia Princiotta Cariddi ${ }^{4,5} \cdot$ Marco Mauri $^{4}$. \\ Fabio Baruzzi ${ }^{1} \cdot$ Maurizio Versino ${ }^{4}$
}

Received: 9 May 2020 / Revised: 13 May 2020 / Accepted: 15 May 2020 / Published online: 2 June 2020

(C) International Spinal Cord Society 2020

\section{To the Editor:}

We read with interest the recent letter to the Editor-in-Chief about SARS-CoV-2 [1]. We wanted to highlight in this letter that although SARS-CoV-2 has a preferential pulmonary and gastrointestinal tropism, it also has systemic and possible neurological effects [2]. We describe the case of a COVID-19 patient presenting to our hospital with newly diagnosed acuteonset tetraparesis in an attempt to increase awareness.

A 22-year-old woman, affected by type I diabetes mellitus, presented to our emergency with worsening dyspnea, fever, and loss of consciousness (Glasgow Coma Scale 6). The first blood investigation showed a state of ketoacidosis ( $\mathrm{pH} 7.05)$, hypocapnia, and hypoxia. Blood glycemia was $744 \mathrm{mg} / \mathrm{dL}$. Table 1 shows blood test trend during patient hospitalization.

A thoracic high-resolution computer tomography (CT) revealed bilateral ground-glass opacity almost completely involving lung parenchyma, highly suggestive for COVID19 pattern. Brain CT and CT-angiography highlighted a tiny right frontal parenchymal hemorrhage, excluding vascular malformations. Subsequently, a reverse transcriptase polymerase chain reaction (RT-PCR) assays of nasopharyngeal swab and bronchoalveolar lavage resulted positive for SARS-CoV-2 infection.

Edoardo Agosti

edoardo_agosti@libero.it

1 Department of Neuroradiology, ASST Sette Laghi, University of Insubria, Varese, Italy

2 Department of Radiology, University of Insubria, Varese, Italy

3 Division of Neurosurgery, Department of Biotechnology and Life Sciences, University of Insubria, Varese, Italy

4 Department of Neurology and Stroke Unit, ASST Sette Laghi, University of Insubria, Varese, Italy

5 Clinical and Experimental Medicine and Medical Humanities, Center of Research in Medical Pharmacology, University of Insubria, Varese, Italy
The patient, already intubated, was then transferred to the intensive care unit. Supportive, antiviral, and immunomodulatory therapies have been set.

On 8th day after hospitalization, a thoracic CT showed the persistence of the ground-glass parenchyma opacity pattern. On 12th day, given the partial improvement of respiratory dynamics and the stability of gas exchange, the endotracheal tube was removed.

On 15th day, a neurological examination highlighted an acute flaccid tetraparesis, more pronounced in the distal muscles, with hyperelicitable osteo-tendon reflexes, and concomitant fecal and urinary incontinence. Occasional and migrant hypoaesthetic and dysaesthetic manifestations have been reported in the lower limbs. On 18th day, brain and spine gadolinium magnetic resonance imaging were performed; no particular pathological features were noticed, except for a late subacute phase tiny frontal hemorrhage ( 8 $\mathrm{mm}$ of maximum diameter).

A lumbar puncture and serology examination were also performed (Table 1). RT-PCR on CSF sample resulted negative for SARS-CoV-2 infection. Physiokinesitherapy rehabilitation was then performed, and a subsequent mild and progressive improvement of hyposthenia and sphincter incontinence was observed.

On 30th day, due to clinical improvement, the patient was discharged and addressed to a rehabilitation facility to complete physiokinesitherapy program.

Since the main organic, infectious, and immunomediated causes of acute-onset flaccid tetraparesis have been excluded from the anamnestic, neuroradiological, and laboratory data recorded, we can hypothesize that SARS-CoV-2 had a promoter role in the onset of the tetraparetic clinical presentation.

Early evidence in the literature has already highlighted how SARS-CoV-2 presents a neurotrophic and neuroinvasive tendency [3]. In particular, through ACE-2 host receptor expressed on the plasma membrane, SARS-CoV-2 can infect different human cells and tissues. By exploiting the same biomolecular mechanisms, SARS-CoV-2 can interact with nervous cells and endothelial cells of the 
Table 1 Patient laboratoristic parameters. This table summarizes patient laboratoristic parameters recorded during hospitalization and subsequent rehabilitation period.

\begin{tabular}{|c|c|c|c|c|c|c|c|c|c|}
\hline & Day 0 & Day 3 & Day 5 & Day 10 & Day 15 & Day 20 & Day 25 & Day 30 & Day 35 \\
\hline \multicolumn{10}{|l|}{ Count $\times 10^{9} / \mathrm{L}$} \\
\hline White blood cells & $28.57 \uparrow$ & $23.99 \uparrow$ & 8.62 & $11.41 \uparrow$ & 9.68 & $11.08 \uparrow$ & 9.63 & 7.00 & \\
\hline Neutrophil & $23.56 \uparrow$ & $21.92 \uparrow$ & $6.96 \uparrow$ & $9.93 \uparrow$ & $7.25 \uparrow$ & 4.03 & 3.85 & 3.98 & \\
\hline Lymphocyte & 3.82 & $1.46 \uparrow$ & $1.12 \uparrow$ & $0.93 \downarrow$ & $0.88 \downarrow$ & $0.82 \downarrow$ & $1.00 \downarrow$ & $0.94 \downarrow$ & \\
\hline Platelet & 365 & 192 & 172 & 313 & 248 & 223 & 260 & 306 & \\
\hline C-reactive protein $\mathrm{mg} / \mathrm{L}$ & $136.1 \uparrow$ & $22.6 \uparrow$ & $10.2 \uparrow$ & 1.9 & 0.7 & 4.5 & 2.0 & 0.9 & \\
\hline D-dimer ug/L & $>9000 \uparrow$ & $3993 \uparrow$ & $3858 \uparrow$ & $4169 \uparrow$ & $2308 \uparrow$ & $902 \uparrow$ & $471 \uparrow$ & 462 & \\
\hline Glucose mg/dL & $744 \uparrow$ & $565 \uparrow$ & $503 \uparrow$ & $476 \uparrow$ & $396 \uparrow$ & $302 \uparrow$ & $273 \uparrow$ & $180 \uparrow$ & \\
\hline Lactate dehydrogenase U/L & $729 \uparrow$ & $618 \uparrow$ & $567 \uparrow$ & $434 \uparrow$ & $360 \uparrow$ & $338 \uparrow$ & $348 \uparrow$ & $222 \uparrow$ & \\
\hline Alanine aminotransferase U/L & 38 & $45 \uparrow$ & 31 & 27 & 40 & $76 \uparrow$ & $69 \uparrow$ & 32 & \\
\hline Aspartate aminotransferase U/L & $144 \uparrow$ & $69 \uparrow$ & 30 & 20 & 28 & $56 \uparrow$ & 29 & 14 & \\
\hline Blood urea nitrogen $\mathrm{mg} / \mathrm{dL}$ & 48 & 31 & 33 & 27 & $16 \downarrow$ & $15 \downarrow$ & 24 & 28 & \\
\hline Creatinine $\mathrm{mg} / \mathrm{dL}$ & 0.64 & 0.46 & 0.33 & $0.29 \downarrow$ & $0.28 \downarrow$ & $0.27 \downarrow$ & $0.29 \downarrow$ & $0.27 \downarrow$ & \\
\hline Cobalamin (B-12) pg/mL & & & & & & 1155 & & & \\
\hline $\begin{array}{l}\text { RT-PCR on nasopharyngeal swab and } \\
\text { bronchoalveolar lavage }\end{array}$ & + & & & & + & & & - & - \\
\hline \multicolumn{10}{|l|}{ Serology } \\
\hline Ig Myc Pne & & & & & - & & & & \\
\hline Ig Leg Pne & & & & & - & & & & \\
\hline Ig Chl Pne & & & & & - & & & & \\
\hline Ig HIV 1-2 & & & & & - & & & & \\
\hline \multicolumn{10}{|l|}{ CSF examination } \\
\hline \multicolumn{10}{|l|}{ Biochemical } \\
\hline Physical & & & & & $\begin{array}{l}\text { Clear, colorless, } \\
\text { normal pressure }\end{array}$ & & & & \\
\hline Glucose & & & & & $139 \mathrm{mg} / \mathrm{dL}$ & & & & \\
\hline Protein & & & & & $53 \mathrm{mg} / \mathrm{dL}$ & & & & \\
\hline Protein & & & & & $0.8 \mathrm{~mm}^{3}$ & & & & \\
\hline \multicolumn{10}{|l|}{ Microscopic } \\
\hline Bacteria & & & & & & - & & & \\
\hline HSV 1-2 DNA & & & & & & - & & & \\
\hline VZV DNA & & & & & & - & & & \\
\hline Mycobacterium & & & & & & - & & & \\
\hline Borrelia & & & & & & - & & & \\
\hline RT-PCR SARS-CoV-2 & & & & & & - & & & \\
\hline
\end{tabular}

+ positive, - negative, $\uparrow$ value higher than laboratory reference parameters, $\downarrow$ value lower than laboratory reference parameters, Chle Pne Chlamydia Pneumoniae, HIV human immunodeficiency virus, HSV Herpes simplex virus, Ig M and G Immunoglobulin classes, Leg Pne Legionella Pneumophila, Myc Pne Mycoplasma Pneumoniae, RT-PCR reverse transcriptase polymerase chain reaction, VZV Varicella zoster virus.

central nervous system (CNS) vessels [3]. Once reached the CNS, SARS-CoV-2 can determine the activation of self-reinforcing inflammatory processes through a "cytokine storm", causing irreversible neuronal damage. In addition, the endothelial ruptures in cerebral capillaries, due to the endotheliitis process, can contribute to the pathophysiology of SARS-CoV-2 brain damage [3].

ACE-2 receptor is also expressed on the plasma membrane of spinal cord neurons [2]. A first-reported case of postinfective acute transverse myelitis has been described in the literature [2], suggesting that spinal cord can be the target of SARS-CoV-2 infection. As for other viruses implicated in postinfectious acute myelitis, the nervous tissue damage is not only given by a direct neuroinvasive action of SARS-CoV-2, but also by an indirect injury of the hyperactivated immune system [3].

It must be underlined that in the case here described, despite the CSF RT-PCR resulted negative for SARS-CoV- 
2 research, the conjunction between neurological clinic, laboratoristic, and thoracic imaging data may support the possible SARS-CoV-2 CNS involvement, confirming its neuroinvasive and neurotrophic character role. Furthermore, the acute onset of a transient tetraparetic status in the absence of neuroradiological evidence of organic damage leads us to support the probable neuro-irritative and neuroshocking role of the SARS-CoV-2.

In addition, our patient suffered from type I diabetes mellitus. As known, the high chronic glucose blood levels can injure nerves throughout different biochemical pathways [4]. Therefore, we can deduce that the neuro-shocking and neuroirritative effect of SARS-CoV-2, in addition to hyperglycemic neuro-stress, can have a promoting and synergistic role in the manifestation of the tetraparetic transient acute clinic.

In summary, while it is well known that SARS-CoV-2 primarily targets pulmonary and gastrointestinal systems, there is now also clear evidence of its systemic involvement. Moreover, early evidence suggests that is may also target the CNS, but we clearly need more data and studies to confirm this. As described in this case, the neuroinvasive and neurotrophic potential of SARS-CoV-2 could have a synergistic and promoter role in determining neuro-irritative and neuro-shocking effects, without necessarily causing neuroimaging evident organic damage. We call on all to be aware of this possibility and to ensure that any cases like this are reported so we can develop a clear picture of all the possible effects of this world-changing virus.

\section{Compliance with ethical standards}

Conflict of interest The authors declare that they have no conflict of interest.

Publisher's note Springer Nature remains neutral with regard to jurisdictional claims in published maps and institutional affiliations.

\section{References}

1. Agosti E, Giorgianni G, Locatelli D. Impact of COVID-19 outbreak on spinal pathology: single center first impression. Spinal Cord. 2020. https://doi.org/10.1038/s41393-020-0480-0.

2. Zhao K, Huang J, Dai D, Feng Y, Liu L, Nie S. Acute myelitis after SARS-CoV-2 infection: a case report. Medrivx. 2020. https://doi. org/10.1101/2020.03.16.20035105.

3. Baig AM, Khaleeq A, Ali U, Syeda H. Evidence of the COVID-19 virus targeting the CNS: tissue distribution, host-virus interaction, and proposed neurotropic mechanisms. ACS Chem Neurosci. 2020;11:995-8. https://doi.org/10.1021/acschemneuro.0c00122.

4. Vinik AI, Nevoret ML, Casellini C, Parson H. Diabetic neuropathy. Endocrinol Metab Clin North Am. 2013;42:747-87. https://doi.org/ 10.1016/j.ecl.2013.06.001. 